\title{
Bloqueo del plano del erector espinal como manejo de dolor neuropático en paciente pediátrico postquemado
}

\section{Erector spinalis plane block as a neuropathic pain management in post-burned pediatric patient}

C. E. Vela Izquierdo¹, M. A. Narváez Tamayo², E. S. Renilla Carranza ${ }^{3}$, M. Fiestas Bancayan4 y M. Rodríguez Calderón ${ }^{4}$

${ }^{\top}$ Médico Anestesiólogo, Máster en Tratamiento del Dolor del Servicio de Centro Quirúrgico y de la Unidad de Terapia del Dolor - Cuidados Paliativos del Instituto Nacional de Salud del Niño de San Borja. Lima, Perú. ${ }^{2}$ Médico Anestesiólogo-Medicina del Dolor, Unidad de Terapia del Dolor y Cuidados Paliativos del Hospital Obrero N¹. Hospital Materno Infantil. Caja Nacional de Salud. La Paz, Bolivia. ${ }^{3}$ Médico Anestesiólogo. Jefa de la Unidad de Terapia del Dolor y Cuidados Paliativos del Instituto Nacional de Salud del Niño de San Borja. Lima, Perú. ${ }^{4}$ Médico Anestesiólogo del Servicio de Centro Quirúrgico y de la Unidad de Terapia del Dolor - Cuidados Paliativos del Instituto Nacional de Salud del Niño de San Borja. Lima, Perú

\section{ABSTRACT}

Pain management is a critical piece in the general care of the burned child. Neuropathic pain is one of its frequent consequences, which may appear at the beginning or during the wound healing process, accompanied by symptoms predominantly due to stimulation of the sympathetic nervous system, its management being an important challenge.

The spinal erector plane [ESP] block is a novel regional technique that has been used in different types of surgery, with promising results. Currently, the ESP block in the pediatric population has been performed for thorax, abdomen, hip and genital surgeries, with only a few reports. As far as we know, ESP block for neuropathic pain in children has not yet been reported. The present report suggests that the thoracic ESP blockade performed at the T4 level could provide a wide and effective analgesia in neuropathic pain in addition to regulating sympathetic symptomatology, secondary to the pathophysiological changes related to the degree of burn.

Key words: Blockade of the spinal erector plane, regional technique, neuropathic pain, burned child, locoregional analgesia.
Vela Izquierdo CE, Narváez Tamayo MA, Renilla Carranza ES, Fiestas Bancayan M, Rodríguez Calderón M. Bloqueo del plano del erector espinal como manejo de dolor neuropático en paciente pediátrico postquemado. Rev Soc Esp Dolor 2020;27(2):127-132

\section{RESUMEN}

El manejo del dolor es una pieza crítica en el cuidado general del niño quemado. El dolor neuropático es una de sus consecuencias frecuentes, el cual puede aparecer al inicio o durante el proceso de cicatrización de heridas, acompañándose de sintomatología predominantemente por estimulación del sistema nervioso simpático, siendo su manejo un reto importante.

El bloqueo del plano del erector espinal (ESP) es una técnica regional novedosa que se ha utilizado en diferentes tipos de cirugía, con resultados prometedores. Actualmente, el bloqueo ESP en la población pediátrica se viene realizando para cirugías de tórax, abdomen, cadera y genitales, con solo pocos informes. Hasta donde sabemos, el bloqueo ESP para dolor neuropático en niños aún no se ha reportado. El presente informe sugiere que el bloqueo ESP torácico realizado a nivel T4 podría proporcionar una analgesia amplia y efectiva en el dolor neuropático además de regular la sintomatología simpática, secundaria a los cambios fisiopatológicos relacionados con el grado de quemadura.

Palabras clave: Bloqueo del plano erector espinal, técnica regional, dolor neuropático, niño quemado, analgesia locorregional.

Recibido: 01-11-2019

Aceptado: 08-01-2020

Correspondencia: César Edwin Vela Izquierdo cevela_izquierdo@hotmail.com 


\section{INTRODUCCIÓN}

Las lesiones por quemaduras en los niños siguen siendo un problema epidemiológico importante en todo el mundo. Casi una cuarta parte de todas las lesiones por quemaduras ocurren en niños menores de 16 años, de los cuales la mayoría son menores de 5. La mayoría de las lesiones por quemaduras son leves y no requieren hospitalización. Una minoría de las lesiones por quemaduras son serias y cumplen con los criterios para ser transferidas a un centro de quemados. El cuidado de estos niños críticos requiere un esfuerzo coordinado y experiencia en el manejo del paciente quemado. La tasa de mortalidad después de quemaduras importantes en estos centros altamente especializados es inferior al $3 \%$. Esto le brinda a un equipo de pediatras, cirujanos, anestesiólogos, intensivistas, enfermeras, terapeutas físicos-respiratorios y otros proveedores de atención médica una oportunidad única de realizar un esfuerzo de colaboración multidisciplinario para ayudar a algunos de los pacientes más vulnerables [1].

La causa de las lesiones por quemaduras varía según la edad del niño. Las lesiones por escaldadura tienden a ser el tipo más común de lesiones térmicas en menores de 5 años, y representan más del $65 \%$ de los casos. Las lesiones por quemaduras importantes no solo provocan daños locales por la lesión incitadora, sino que en muchos casos resultan en manifestaciones sistémicas, secundario a la liberación de marcadores inflamatorios, mediadores vasoactivos, catecolaminas, pérdida de proteínas y otros mecanismos. Los esfuerzos iniciales se centran en la reanimación, el mantenimiento de la estabilidad hemodinámica y el manejo de las vías respiratorias. Los esfuerzos intermedios se centran en manejar la falla multiorgánica. Finalmente, los esfuerzos cambian a problemas con la curación de heridas crónicas, el manejo del dolor, la restauración de las capacidades funcionales y la rehabilitación (1).

El manejo del dolor es una pieza crítica en el cuidado general del niño quemado. El dolor intenso es una consecuencia importante de la lesión por quemadura, y se ha demostrado que a menudo no se trata adecuadamente. La ansiedad y la depresión son componentes de confusión en una quemadura mayor y pueden disminuir aún más el umbral del dolor. Debido a las preocupaciones de tolerancia, abstinencia e hiperalgesia inducida por opioides, se ha recomendado el uso de un régimen de control del dolor multimodal. Las técnicas anestésicas regionales también pueden servir como un complemento útil para ahorrar opioides [2].

El dolor crónico en pacientes quemados tiene orígenes múltiples y a menudo poco claros. El dolor neuropático es una de las causas de este y se desarrolla como consecuencia del daño nervioso, anomalías en la regeneración nerviosa y la reprogramación del sistema nervioso central (3). El prurito severo ocurre en hasta el $87 \%$ de los pacientes adultos y el $100 \%$ de los pacientes con quemaduras pediátricas durante el proceso de curación. Tiende a presentarse de forma temprana durante la cicatrización de heridas y continúa mucho después de la reepitelización y la maduración de la cicatriz, y puede afectar significativamente la calidad de vida. Se ha utilizado una variedad de terapias, tanto sistémicas como tópicas, para el tratamiento del prurito posquemadura, también llamado prurito neuropático, por su relación con las fibras nerviosas tipo $C$ (4).

El bloqueo del plano del erector espinal, o ESP por sus siglas en inglés, es una técnica relativamente reciente (5) y simple que sugiere ser prometedora y ciertamente segura en la analgesia torácica tanto en el dolor neuropático crónico como en el dolor posquirúrgico. La inyección en esta región muestra diseminación del medicamento administrado en al menos 4 niveles vertebrales por encima y 3 niveles vertebrales por debajo del punto de inyección en dirección cefalocaudal (6), paravertebral e incluso hasta el origen de los nervios intercostales y ramos dorsales, lo que resulta en una muy buena analgesia del hemitórax en su pared lateral, anterior y posterior. El anestésico local también penetra anteriormente, a través del tejido conectivo intertransverso y entra en el espacio paravertebral torácico, donde puede bloquear potencialmente no solo las ramas ventrales y dorsales de los nervios espinales, sino también las ramas comunicantes que transmiten fibras simpáticas [7], lo que ofrece una explicación lógica para los amplios cambios sensoriales y analgésicos hallados posterior al procedimiento. Teniendo en cuenta que hasta la actualidad no se encuentran reportes de casos de bloqueo ESP en dolor neuropático para paciente pediátrico, decidimos realizarlo, puesto que agotamos todas las medidas farmacológicas y no farmacológicas, y basándonos en la teoría y estudios realizados en pacientes pediátricos para otras patologías.

\section{DESCRIPCIÓN DEL CASO}

Paciente de sexo femenino, de 2 años, con $15 \mathrm{~kg}$ de peso, remitido al hospital por quemaduras del $40-49 \%$ producidas por agua caliente, comprometiendo cara, tronco anterior, tronco posterior y miembros superiores. La paciente requirió ingreso en la Unidad de Cuidados Intensivos de Quemados (UCIQM), en donde se solicita interconsulta desde el ingreso a la Unidad de Terapia del Dolor y Cuidados Paliativos (UTDCPP) para optimizar manejo analgésico. En el momento de la valoración inicial, se encuentra al paciente con un FLACC [Face, Legs, Activity, Cry, Consolability] de 4 puntos [0 a 10 puntos] [8], iniciando administración de tramadol a $1 \mathrm{mg} / \mathrm{kg}$ vía intravenosa [i.v. ] $/ 6 \mathrm{~h}$, metamizol $25 \mathrm{mg} / \mathrm{kg}$ i.v. $/ 6 \mathrm{~h}$ y morfina 1,5 mg i.v. condicional a dolor intenso. Durante su estancia en UCIQM, ingresa a sala de operaciones para realización de limpiezas quirúrgicas, xenoinjertos, autoinjertos dermoepidérmicos de espesor parcial y curaciones, con las cuales el dolor se exacerba después de los procedimientos quirúrgicos mencionados, asociándose además prurito de moderada a severa intensidad en las regiones operadas [9]. Por ello se decide suspender tramadol e iniciar analgesia controlada por el paciente (PCA) de morfina más ketamina en concentración de $0,5 \mathrm{mg} / \mathrm{ml}$ de ambos medicamentos con una infusión que variaba entre 0,6 ml hasta 0,8 ml/h, bolos de 0,5-1 ml, bloqueo de 10 min y $2-4$ bolos/h (en este caso los bolos eran controlados por la enfermera [NCA]) (10); además gabapentina hasta $40 \mathrm{mg} / \mathrm{kg} /$ día y bolos horarios de 


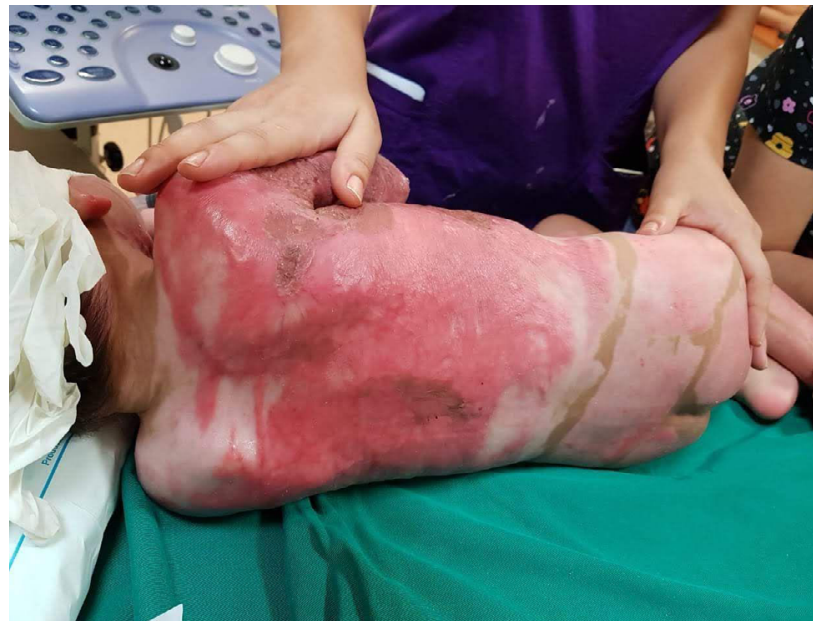

Fig. 1. Secuela de quemadura, ingresando para curación.

lidocaína más ketamina, de 1-2 mg/kg del primero y $0,1-0,2 \mathrm{mg} / \mathrm{kg}$ del segundo medicamento. Posteriormente la paciente es trasladada a hospitalización de pacientes quemados, en donde le continúan realizando curaciones interdiarias (Figura 1). Durante la evaluación diaria por el equipo de la UTDCPP se encuentra un dolor controlado (FLACC 1 a 2 puntos) y persistencia de prurito de severa intensidad en tórax anterior y posterior bilateral, que le impedía la conciliación del sueño, la realización de actividades simples y la colocación de prendas o ropas, decidiendo suspender PCA e iniciar tramadol de 1-1,5 mg/kg horario (cada 6-24 h y la suspensión respectiva), se adiciona amitriptilina de 0,25-0,8 mg/kg/dia, se suspende bolos de lidocaína/ketamina y se inicia infusión de lidocaína de $1-1,5 \mathrm{mg} / \mathrm{kg} / \mathrm{h}$ además de loratadina y clorfenamina horaria, indicada por su médico pediatra tratante. Al realizar el seguimiento respectivo de la paciente se evidencia un FLACC de 1 punto y prurito de severa intensidad en las mismas regiones, decidiendo continuar con la medicación antes descrita e iniciar sesiones de acupuntura, remitiendo parcialmente el prurito. Por ello se plantea en junta médica la realización de una técnica analgésica regional. Teniendo en cuenta que la región afectada era muy amplia y por el riesgo considerable de neumotórax, se descarta el bloqueo de nervios intercostales. Igualmente, la inyecsción torácica epidural o paravertebral se descartó, al considerarse muy invasiva. Finalmente, por su efecto descrito a nivel nociceptivo, neuropático y simpático, se optó por un bloqueo del ESP guiado por ultrasonido, según la técnica descrita por Forero y cols. [5]. Paciente bajo sedación, con funciones vitales estables, quien presenta una piel hipertrófica, eritematosa, sin signos o síntomas de infección local, se ubica en posición lateral; usando el transductor lineal de alta frecuencia (5-15 Mhz) se visualiza inicialmente en eje largo la apófisis espinosa de T4, posteriormente se giró el transductor al eje corto, se identificaron 3 músculos superficiales (trapecio, romboide mayor y erector espinal] y con una aguja espinal punta quincke $\mathrm{BD}^{\circledR}$ calibre $22 \mathrm{G} \times 3 \frac{1}{2}$ p pulgadas, se hace la punción en plano con el transductor, en dirección

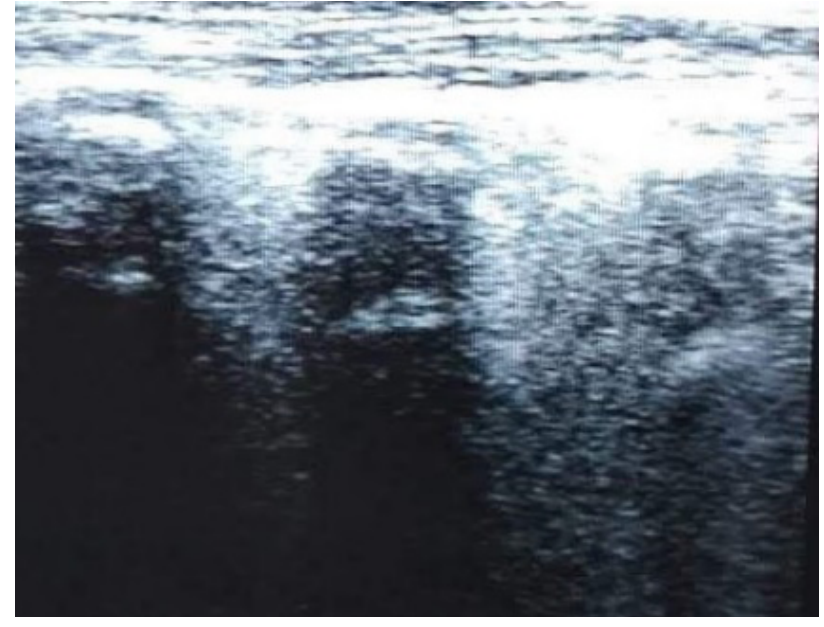

Fig. 2. Imagen ecográfica en el plano del erector espinal.

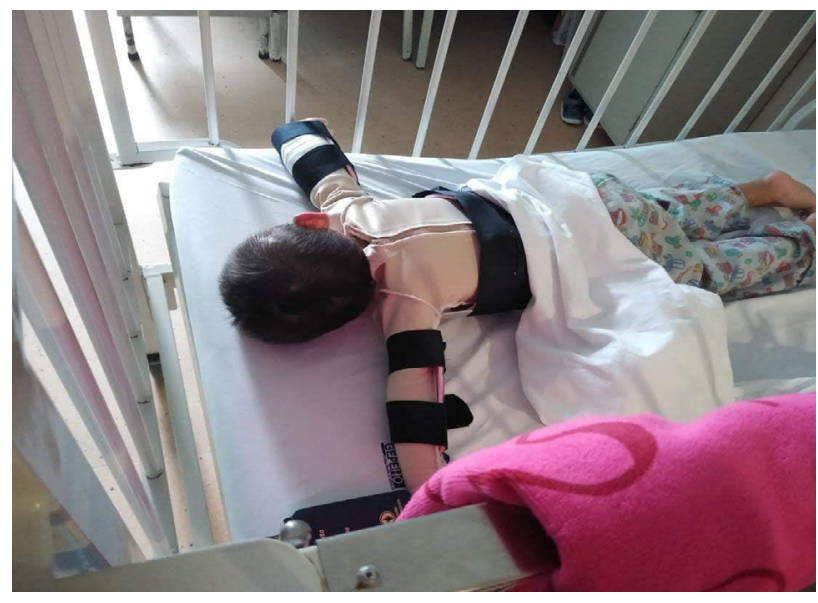

Fig. 3. Paciente con uso de prenda elástica y ropa, con adecuada conciliación del sueño.

cefalocaudal buscando el plano interfascial superficial a las apófisis transversa de T4. Con ultrasonido se localiza la punta de la aguja en el plano interfascial y se inyecta un total de $0,5 \mathrm{ml} / \mathrm{kg}$ de bupivacaína al 0,1\% más dexametasona $0,1 \mathrm{mg} / \mathrm{kg}$, bilateral (Figura 2); el cual se realizó en 3 oportunidades, espaciados por 3 días.

Se hizo seguimiento diario, evidenciando mejoría considerable de prurito, hasta la ausencia de dicho síntoma, permitiendo el uso de ropa, mejoría del sueño, sesiones de terapia física, realización de actividades básicas y jugar (Figura 3). Al mismo tiempo se decide retirar paulatinamente los medicamentos administrados con anterioridad, quedando solo con gabapentina, brindándose posteriormente el alta respectiva. Luego es evaluada por consultorio externo de terapia del dolor, presentando ausencia de prurito, tolerando las sesiones de terapia física y el uso de prenda elástica, decidiendo el retiro completo de gabapentina y el alta del servicio. 


\section{DISCUSIÓN}

El manejo del dolor es una pieza crítica en el cuidado general del niño quemado, recomendándose el uso de un régimen de control del dolor multimodal. Las técnicas anestésicas regionales también pueden servir como un complemento útil para ahorrar opioides, evitar su consumo y los eventos adversos de los mismos.

El dolor neuropático es uno de los problemas frecuentes en un niño quemado, se desarrolla como consecuencia del daño nervioso, anomalías en la regeneración nerviosa y la reprogramación del sistema nervioso central, el cual puede aparecer al inicio o durante el proceso de cicatrización de heridas, acompañándose de cambios simpáticos, por lo que su manejo es un reto importante.

El bloqueo del ESP es una técnica recientemente descrita en pacientes adultos y para manejo de dolor neuropático, según mencionado por los autores [5].
Actualmente el bloqueo ESP se viene realizando en la población pediátrica, con solo pocos informes (Tabla I), para manejo del dolor postoperatorio de cirugías torácicas $(11-15,20,21)$, nefrectomía (16), reparación de hernia inguinal (17), colecistectomía laparoscópica (18), displasia de cadera (19) e hipospadias (22). Hasta donde sabemos, el bloqueo ESP para dolor neuropático en niños aún no se ha reportado.

Forero y cols. [5] informaron que en el bloqueo ESP el anestésico local se disemina al menos 4 niveles vertebrales por encima y 3 niveles vertebrales por debajo del punto de inyección en dirección cefalocaudal, además penetra anteriormente a través del tejido conectivo intertransverso, obteniendo acceso indirecto al espacio paravertebral donde puede bloquear potencialmente las ramas dorsal y ventral de los nervios espinales. Del mismo modo Chin y cols. (7) mencionaron que también puede bloquear las fibras nerviosas simpáticas. Basándonos en lo descrito anteriormente, el presente informe

TABLA I

CASOS PUBLICADOS DE BLOQUEO DEL PLANO ERECTOR ESPINAL EN LA POBLACIÓN PEDIÁTRICA

\begin{tabular}{|c|c|c|c|c|c|c|}
\hline Autor & Edad & Indicación & Nivel & AL y concentración & Volumen & $\begin{array}{c}\text { Difusión } \\
\text { del AL }\end{array}$ \\
\hline $\begin{array}{l}\text { Muñoz y cols. } \\
\text { (11) }\end{array}$ & 7 años & $\begin{array}{l}\text { Tumor de la } \\
\text { undécima costilla } \\
\text { derecha }\end{array}$ & Т 8 & Bupivacaína 0,5 \% & $\begin{array}{l}14 \mathrm{ml} \text { (no } \\
\text { precisa por } \\
\text { peso) }\end{array}$ & T5-T11 \\
\hline $\begin{array}{l}\text { De la Cuadra- } \\
\text { Fontaine y cols. } \\
\text { (12) }\end{array}$ & 3 años & $\begin{array}{l}\text { Cirugía abierta de } \\
\text { tórax }\end{array}$ & T9 & Levobupivacaína 0,25 \% & $0,6 \mathrm{ml} / \mathrm{kg}$ & NP \\
\hline $\begin{array}{l}\text { Hernández y } \\
\text { cols. (13) }\end{array}$ & 3 años & $\begin{array}{l}\text { Lipoma paraespinal } \\
\text { torácico }\end{array}$ & T1 & $\begin{array}{l}\text { Bupivacaína 0,25 \% y } \\
\text { lidocaína } 1 \%\end{array}$ & $0,2 \mathrm{ml} / \mathrm{kg}$ & NP \\
\hline $\begin{array}{l}\text { Ueshima y } \\
\text { Otake [14] }\end{array}$ & $\begin{array}{l}6 \text { años } \\
8 \text { años } \\
\end{array}$ & Pectus excavatum & T6 (BB) & Levobupivacaína 0,25 \% & \begin{tabular}{|l}
$0,6 \mathrm{ml} / \mathrm{kg}$ (en \\
cada lado)
\end{tabular} & NP \\
\hline $\begin{array}{l}\text { Kaplan y cols. } \\
\text { (15) }\end{array}$ & 7 meses & $\begin{array}{l}\text { Lobectomía } \\
\text { superior izquierda }\end{array}$ & T6 & $\begin{array}{l}\text { B: ropivacaína 0,2 \% } \\
\text { I: } \text { ropivacaína 0,2 \% }\end{array}$ & $\begin{array}{l}0,3 \mathrm{ml} / \mathrm{kg} \\
0,15 \mathrm{ml} / \mathrm{kg} / \mathrm{h}\end{array}$ & T3-Т10 \\
\hline $\begin{array}{l}\text { Aksu y Gurkan } \\
\text { (16) }\end{array}$ & $\begin{array}{l}7 \text { años } \\
6 \text { meses }\end{array}$ & Nefrectomía & T12 & Bupivacaína 0,25 \% & $0,5 \mathrm{ml} / \mathrm{kg}$ & NP \\
\hline $\begin{array}{l}\text { Hernández y } \\
\text { cols. [17] }\end{array}$ & 2 meses & Hernia inguinal & T6 & $\begin{array}{l}\text { Bupivacaína 0,25 \% y } \\
\text { lidocaína } 1 \%\end{array}$ & $0,4 \mathrm{ml} / \mathrm{kg}$ & T4-L1 \\
\hline $\begin{array}{l}\text { Thomas y } \\
\text { Tulgar } 18]\end{array}$ & 11 años & $\begin{array}{l}\text { Colecistectomía } \\
\text { laparoscópica }\end{array}$ & T9 & Bupivacaína 0,25 \% & $0,6 \mathrm{ml} / \mathrm{kg}$ & NP \\
\hline $\begin{array}{l}\text { Elkoundi y cols. } \\
\text { (19) }\end{array}$ & 4 años & \begin{tabular}{|l} 
Displasia del \\
desarrollo de \\
cadera
\end{tabular} & L2 & Bupivacaína 0,25 \% & $0,3 \mathrm{ml} / \mathrm{kg}$ & L1-L4 \\
\hline $\begin{array}{l}\text { C. Gaio-Lima y } \\
\text { cols. (20) }\end{array}$ & 15 meses & $\begin{array}{l}\text { Resección tumoral } \\
\text { por toracotomía }\end{array}$ & T5 & $\begin{array}{l}\text { B: ropivacaína 0,2 \% } \\
\text { I: ropivacaína 0,1\% }\end{array}$ & $\begin{array}{l}0,45 \mathrm{ml} / \mathrm{kg} \\
2 \mathrm{ml} / \mathrm{h}\end{array}$ & T1-T8 \\
\hline $\begin{array}{l}\text { Patel y cols. } \\
\text { [21] }\end{array}$ & 6 años & $\begin{array}{l}\text { Lobectomía inferior } \\
\text { derecha }\end{array}$ & T5 & $\begin{array}{l}\text { B: ropivacaína 0,5 \% } \\
\text { I: ropivacaína 0,1\% }\end{array}$ & $\begin{array}{l}10 \mathrm{ml}(0,5 \mathrm{ml} / \\
\mathrm{kg}) \\
0,2 \mathrm{mg} / \mathrm{kg} / \mathrm{h}\end{array}$ & NP \\
\hline $\begin{array}{l}\text { Aksu y cols. } \\
\text { [2己] }\end{array}$ & 6 meses & Hipospadias & S4 & Bupivacaína 0,25 \% & $1 \mathrm{ml} / \mathrm{kg}$ & NP \\
\hline
\end{tabular}

AL: anestésico local. NP: no precisa. BB: bloqueo bilateral. B: bolo, I: infusión 
sugiere que el bloqueo torácico ESP realizado a nivel T4 podría proporcionar una analgesia amplia y efectiva en el dolor neuropático además de regular la sintomatología simpática, secundaria a los cambios fisiopatológicos relacionados con el grado de quemadura, siendo además el uso del ultrasonido una herramienta de profundo peso para el médico intervencionista, puesto que ofrece no solo seguridad clínica al paciente, sino que en otro sentido corrobora la efectividad de la técnica seleccionada.

Hasta la fecha, no hay recomendaciones sobre la dosis óptima de anestésico local para usar en un bloqueo ESP en niños. En algunos informes anteriores, se utilizaron volúmenes relativamente más pequeños de anestésico local o volúmenes y concentraciones similares o superiores a los informados en este caso. De la Cuadra y cols. (12) utilizaron un volumen de $0,6 \mathrm{ml} / \mathrm{kg}$ de levobupivacaína al 0,25\% para realizar un bloqueo ESP analgésico en cirugía de tórax. Ueshima y Otake (14) informaron el uso exitoso de levobupivacaína al $0,25 \%$ con un bolo de 0,6 ml/kg bilateral, en un niño de 6 años postoperado de pectum excavatun. Aksu y Gurkan (16) utilizaron $0,5 \mathrm{ml} / \mathrm{kg}$ de bupivacaína al $0,25 \%$ como analgesia postoperatoria de nefrectomía en 2 niños de 7 años y 6 meses. Del mismo modo, Thomas y Tulgar (18) usaron 0,6 ml/kg de bupivacaína al 0,25 \% para colecistectomía laparoscópica y Patel y cols. [21) utilizaron $0,5 \mathrm{ml} / \mathrm{kg}$ de ropivacaína al 0,5\% como analgesia en cirugía de tórax. Los estudios futuros sobre el volumen, la concentración de anestesia local óptima y adyuvantes al realizar el bloqueo ESP en niños serían útiles para los profesionales y para mejorar aún más la comprensión de la utilidad de este bloqueo.

\section{CONCLUSIONES}

El bloqueo del plano del erector espinal es una técnica recientemente descrita en pacientes adultos y para manejo de dolor neuropático. Actualmente el bloqueo ESP se viene realizando en la población pediátrica, con solo pocos informes. En este bloqueo el anestésico local se disemina al menos 4 niveles vertebrales por encima y 3 niveles vertebrales por debajo del punto de inyección en dirección cefalocaudal, además penetra anteriormente a través del tejido conectivo intertransverso, obteniendo acceso indirecto al espacio paravertebral donde puede bloquear potencialmente las ramas dorsal y ventral de los nervios espinales, y las fibras nerviosas simpáticas. Basándonos en lo descrito anteriormente, el presente informe sugiere que el bloqueo torácico ESP realizado a nivel T4 podría proporcionar una analgesia amplia y efectiva en el dolor neuropático además de regular la sintomatología simpática, secundaria a los cambios fisiopatológicos relacionados con el grado de quemadura, del mismo modo reduce el consumo de opioides y sus efectos adversos.

\section{CONFLICTOS DE INTERESES}

Los autores declaran no tener ningún conflicto de intereses.

\section{FUENTES DE FINANCIACIÓN}

Los autores no recibieron patrocinio para llevar a cabo este artículo.

\section{BIBLIOGRAFÍA}

1. Krishnamoorthy V, Ramaiah R, Bhananker S. Pediatric burn injuries. Int J Crit III Inj Sci. 2012;2(3):128-34. DOI: 10.4103/2229-5151.100889.

2. Cuignet O, Pirson J, Soudon O, Zizi M. Effects of gabapentin on morphine consumption and pain in severely burned patients. Burns. 2007;33(1):81-6. DOI: 10.1016/j. burns.2006.04.020

3. Gandhi M, Thomson C, Lord D, Enoch N. Management of pain in children with burns. Int J Pediatr. 2010;2010:1-9. DOI: 10.1155/2010/825657.

4. Goutos I, Dziewulski P, Richardson PM. Pruritus in burns: review article. J Burn Care Res. 2009;30(2):221. DOI: 10.1097/BCR.Ob013e318198a2fa.

5. Forero M, Adhikary SD, Lopez H, Tsui C, Chin KJ. The erector spinae plane block. Reg Anesth Pain Med. 2016;41(5):6217. DOI: 10.1097/AAP.0000000000000451.

6. Vargas J, Vela C, Ricaurte L, Castillo O, Aparicio A. Bloqueo del plano del erector espinal como analgesia en fracturas costales múltiples unilaterales. unilaterales. Rev Soc Esp Dolor. 2019;26(3):199-202.

7. Chin KJ, Malhas L, Perlas A. El bloqueo del plano erector de la columna vertebral proporciona analgesia abdominal visceral en cirugía bariátrica: un informe de 3 casos. Reg Anesth Pain Med. 2017;42(3):372-6. DOI: 10.1097/ AAP.0000000000000581.

8. Merkel S, Voepel-Lewis T, Shayevitz J, Malviya S. The FLACC: a behavioral scale for scoring postoperative pain in young children. Pediatr Nurs. 1997;23(3):293-7.

9. Everett T, Parker K, Fish J, Pehora C, Budd D, Kelly C, et al. The construction and implementation of a novel Postburn Pruritus Scale for infants and children aged five years or less: introducing the Toronto Pediatric Itch Scale. J Burn Care Res. 2014;36(1): 44-9. DOI: 10.1097/ BCR.0000000000000129.

10. James P, Howard R, Glyn D. The addition of ketamine to a morphine nurse- or patient-controlled analgesia infusion (PCA/NCA) increases analgesic efficacy in children with mucositis pain. Pediatr Anaesth. 2010;20(9):805-11. DOI: 10.1111/j.1460-9592.2010.03358.x.

11. Muñoz F, Cubillos J, Bonilla AJ, Chin KJ. Erector spinae plane block for postoperative analgesia in pediatric oncological thoracic surgery. Can J Anaesth 2017;64(8):880-2. DOI: 10.1007/s12630-017-0894-0

12. De la Cuadra-Fontaine JC, Concha M, Vuletin F, Arancibia H. Continuous erector spinae plane block for thoracic surgery in a pediatric patient. Paediatr Anaesth. 2018;28(1):74-5. DOI: 10.1111/pan. 13277 .

13. Hernandez MA, Palazzi L, Lapalma J, Forero M, Chin KJ. Erector spinae plane block for surgery of the posterior thoracic wall in a pediatric patient. Reg Anesth Pain Med. 2018:43(2):217-9.

14. Ueshima $\mathrm{H}$, Otake $\mathrm{H}$. Clinical experiences of erector spinae plane block for children. J Clin Anesth. 2018;44:41. DOI: 10.1016/j.jclinane.2017.10.021

15. Kaplan I, Jiao Y, AuBuchon JD, Moore RP. Continuous erector spinae plane catheter for analgesia after infantthoraco- 
tomy: a case report. A A Pract. 2018;11(9):250-2. DOI: 10.1213/XAA.0000000000000799.

16. Aksu C, Gürkan Y. Ultrasound guided erector spinae block for postoperative analgesia in pediatric nephrectomy surgeries. J Clin Anesth. 2018;45:35-6. DOI: 10.1016/j.jclinane.2017.12.021.

17. Hernandez MA, Palazzi L, Lapalma J, Cravero J. Erector spinae plane block for inguinal hernia repair in preterm infants. Paediatr Anaesth. 2018;28(3):298-9. DOI: 10.1111/ pan. 13325.

18. Thomas DT, Tulgar S. Ultrasound-guided erector spinae plane block in a child undergoing laparoscopic cholecystectomy. Cureus. 2018;10(2):2241.

19. Elkoundi A, Bentalha A, El Kettani S, Mosadik A, Koraichi $A E$. Erector spinae plane block for pediatric hip surgery -a case report-. Korean J Anesthesiol. 2019;72(1):68-71. DOl: 10.4097/kja.d.18.00149.

20. Gaio-Lima C, Costa CC, Moreira JB, Lemos TS, Trindade HL. Bloqueo continuo en el plano del músculo erector del espinal para analgesia en cirugía torácica pediátrica: informe de un caso. Rev Esp Anestesiol Reanim. 2018;65(5):287-90. DOl: 10.1016/j.redar.2017.11.010.

21. Patel N, Glover C, Adler A. Erector spinae plane catheter for postoperative analgesia after thoracotomy in a pediatric patient: a case report. A A Pract. 2019;12(9):299-301. DOl: 10.1213/XAA.0000000000000914.

22. Aksu C, Gürkan Y. Sacral Erector Spinae Plane Block with longitudinal midline approach: Could it be the new era for pediatric postoperative analgesia? J Clin Anesth. 2020; 59:38-9. DOI: 10.1016/j.jclinane.2019.06.007. 